\title{
INTEROPERABILIDADE MÓVEL: A INTERNET DAS COISAS
}

\author{
Wander de Moraes PAES
}

\begin{abstract}
*Mestre em Tecnologias da Inteligência e Design Digital - PUC/SP, Doutorando em Tecnologias da Inteligência e Design Digital - PUC/SP, Consultor de Tecnologia e Gestor de Projetos, Professor COGEAE PUC/SP, Professor IED (Instituto Europeu de Design / SP), wanderpaes@yahoo.com.br
\end{abstract}

\section{Recebido em: 30/05/2014 - Aprovado em: 30/06/2014 - Disponibilizado em: 30/07/2014}

\begin{abstract}
Resumo
A Internet das Coisas relaciona assuntos à comunicação e da troca das informações dos sistemas no ambiente pervasivo por meio da tecnologia móvel, representando a dinâmica que envolve a colaboração a integração no ambiente distribuído do meio digital utilizada pelas soluções sistêmicas nos diversos setores da economia e consequentemente pela sociedade de forma cada vez mais intensa. O que caracteriza o aspecto hibrido, multidisciplinar e interdisciplinar no processo de inovação tecnológica. De modo que a tecnologia sem fio afirma-se como nova tendência de uso dessas soluções devido à popularização dos dispositivos eletrônicos (celular, Tablets, Smartphone, eletroeletrônicos domésticos inteligentes, e da interoperabilidade proporcionada pela Internet) e principalmente pelo papel da sociedade numa caracterização Cíbrida $^{1}$ como parte integrante no processo dinâmico de colaboração das informações. A Internet e a tecnologia móvel, sob a perspectiva da colaboração das informações como uma plataforma para inovação, influenciam diretamente a concepção das soluções no mercado. Esse novo mapa dos serviços descentralizados no Ciberespaço representa também a introdução das mídias móveis e seu reflexo com impacto significativo nas relações humanas e nas soluções sistêmicos comerciais enquadrados no fenômeno da Economia Criativa. Portanto, os assuntos desse artigo representa a ideia da interoperabilidade dos dispositivos móveis sob o contexto dos fenômenos contemporâneos da mobilidade, do Ciberespaço, dos aspectos tecnológicos das redes sem fio, e da arquitetura de sistemas móveis. O que traduz numa apresentação abrangente das relações tecnológicas sobre a influência da mobilidade na sociedade. Sobretudo, enquadra a ideia da Interoperabilidade de uma maneira abrangente, e consequentemente revela o meio digital móvel como parte integrante de uma mesma inovação tecnológica para a colaboração das informações e integração no meio digital.
\end{abstract}

Palavras Chave: Computação Pervasiva/Ubiqua, Mobilidade, Internet das Coisas, Inovação Tecnológica, Interoperabilidade..

\begin{abstract}
The concept about sharing and changing information using Internet resources, the users has been gradually extending the services to integrate, step by step, more technology into their way of life's. The ubiquity introduced variety possibilities of using mobile devices as key of sharing information among peoples in motion and anywhere. In this point of view I present the main challenge in pervasive/ubiquitous concepts for wireless applications networks, focusing on the resource of systems interoperability and information. The idea about interoperability involves the relationship and change of information among different areas, as instrument of dissemination and production of the knowledge into digital environment also applied to the dynamic environment of the mobile devices. So that, the subject in this article remain the necessity of a study to relate the interoperability as a way to the communication and the exchange information among peoples. This phenomenon shows up as a trend of the systems solutions integrated with WEB applications, and now in Wireless and Cellular network used by the electronic devices like Cellular, Smartphone, Tablets, NetBooks, Netbook's, etc. The consolidation of the Internet as a collaboration environment together with the popularity of the mobile applications had directly influenced the business solutions and the systems architecture in the contemporary society. The dynamic of changing information using wireless electronic devices became a new phenomenal inside the cartographic map of the decentralized services in cyberspace named "Internet of Things. These context had influenced the companies and the society in the digital culture, as well all players all over the communication chain in cyberspace.
\end{abstract}

Keywords: Pervasive/Ubiquitous Computing, Mobile, Information Architecture, Interoperability, Innovation Technology.

1 O termo Cíbrido, com base no desenvolvimento dos dispositivos, como a criação de novas realidades são metamorfizadas pelas tecnologias que permitem ao corpo, ampliar, conectar, plugar, reprojetar, remodelar, manipular, imergir, simular, digitalizar, transformando-se o corpo numa hibridação do biológico com o digital (biocibernético). Uma visão do mundo ampliada, reformulada e integrada pelas experiências do corpo em simbiose com as tecnologias (SANTAELLA, 2003). 


\section{Introdução}

A abordagem desse artigo tem como objetivo a representação do Ambiente Informacional tecnológico para contextualizar e servir como referencia aos profissionais dos diversos setores que utilizam ou estão inseridos no processo de inovação para o desenvolvimento de produtos e serviços no meio digital por meio da tecnologia sem fio.

Dessa forma, no desenvolvimento dos assuntos desse artigo, apresenta a contextualização do ambiente sociotecnológico que a o fenômeno da Mobilidade se relaciona com os usuários no Ciberespaço para colaboração das informações. Posteriormente, na seção Tecnologia, relaciona os conceitos relevantes no que tange os recursos de comunicação onde as soluções sistêmicas interoperam as informações caracterizada pelo fenômeno da Internet das Coisas.

$\mathrm{Na}$ seção Resultado uma referência como parte do diagnóstico do projeto de pesquisa de Doutorado voltado para o desenvolvimento das diretrizes de gestão em conjunto dos cenários de mercado que um profissional da área de tecnologia se depara quando engajado no desenvolvimento de produtos e serviços no meio digital. Cenários que já se apresentam aplicados em diversas soluções de negócio e com ampla possibilidade de aplicação das funcionalidades com objetivo de integrar/colaborar informações. E finalmente na seção Conclusão com as considerações e sob a ótica multidisciplinar sobre contexto socio-tecnológico de interoperabilidade informacional no meio móvel pelos usuários.

\section{Desenvolvimento}

\subsection{Ambiente Informacional}

O Ambiente Informacional representa uma abordagem do contexto sóciotecnológico sobre as práticas da integração e colaboração das informações com a rede Internet por meio das ferramentas tecnológicas, com foco nos fenômenos da Mobilidade, Arquitetura da Informação, e da infraestrutura da comunicação sem fio. O que se intitulou como Internet das Coisas.

\section{Simetria e Assimetria no Ambiente Digital}

Inicialmente a Internet foi concebida na topologia P2P (peer-to-peer), possibilitando uma integração ponto a ponto dos indivíduos e serviços por meio da rede. Pressupunha-se que os computadores estivessem sempre ligados e conectados com o endereçamento IP (Internet Protocol) fixo.

Desse modo que a conexão entre os pontos tivesse uma conexão direta e simétrica, permitindo uma proporção equilibrada no fluxo de dados entre o recebimento e o envio das informações. Proporcionando maior colaboração e integração direta com os usuários. A popularização da Internet levou a uma tendência assimétrica, representada pela 
desproporcionalidade do fluxo das informações mediada pelos grandes sites; fazendo da utilização da Internet um exercício maior de recebimento em detrimento ao envio das informações, comprometendo o viés de colaboração da arquitetura original da Internet, conforme Andy Oram (2001, p.21).

A Internet começou como uma rede P2P, totalmente simétrica, de usuários em cooperação. Quando a Rede cresceu de modo a conciliar os milhões de pessoas on-line, as tecnologias existentes a dividiram em um sistema com relativamente poucos servidores e muitos clientes. Ao mesmo tempo, algumas das expectativas básicas da cooperação estão apresentando o risco de ruírem, ameaçando a estrutura da rede. Esse fenômeno impõe desafios e obstáculos aos aplicativos P2P: tanto a rede quanto os aplicativos precisam ser concebidos em conjunto para trabalharem em série. Autores de aplicativos devem projetá-los para que sejam resistentes e possam funcionar no ambiente complexo da Internet,e os projetistas da rede devem incorporar recursos para lidar com aplicativos peer-to-peer. Felizmente, muitas dessas questões são familiares, devido à experiência da antiga Internet; e as ações aprendidas ali podem ser aproveitadas em sistemas que venham a ser aproveitadas no futuro.

Não está em questão uma abordagem crítica em relação à assimetria das redes, pois há movimentos que permitem o equilíbrio da dinâmica informacional por meio da simetria na troca das informações como exemplos das Comunidades Virtuais, BLOGs,E-Mails, em conjunto com aplicativos móveis embarcados. A ideia é elucidar os aspectos na relação
Individuo $\mathrm{x}$ Grupo que representa uma tendência da utilização do meio digital móvel com desdobramentos no contexto social e tecnológico. Uma liberdade para a colaboração das informações e utilização democrática da tecnologia, remontando, por meio da troca das informações pelos aplicativos embarcados nos dispositivos móveis, a ideia e topologia Peer-to-Peer (P2P) precursora da rede Internet.

Entretanto, também não estão exclusivamente em questão os fatores que motivaram o contexto assimétrico predominante da Internet atual como o aumento da largura de banda; centralização dos sites provedores de conexão; ou pressões comerciais que levaram aos moldes na grande maioria da arquitetura informacional da rede que percebemos atualmente. $\mathrm{O}$ foco está relacionado ao ambiente dos dispositivos móveis como uma alternativa no contexto da simetria das redes, remontando a idéia Peerto-Peer (P2P) inicial da Internet, apresentando uma cartografia multidimensional e distribuída das informações. O sinal desta evolução vem se consolidando desde o surgimento das redes corporativas privadas on-line, passando pela popularização da Internet até a emergência da tecnologia dos dispositivos móveis e redes sem fio (WiFi/Wi-Max) baseada numa arquitetura de rede móvel distribuída voltada à colaboração das informações e mobilidade dos indivíduos. Essa configuração do meio digital representa uma relação direta ou indireta com a 
centralização ou descentralização das informações, apresentando cenários que ora corroboram com a centralização e ora com a descentralização. De modo que a utilização do meio caracteriza a representação de um ambiente fértil para implementação de ambos os cenários.

A proposta de liberdade da troca de informações baseada na descentralização e conectividade da arquitetura $\mathrm{P} 2 \mathrm{P}$ (ponto a ponto), proposta pelo ambiente descentralizado, está sob a administração das corporações que são detentoras da tecnologia e infraestrutura das redes sem fio; sites; meios de comunicação e fabricação dos dispositivos eletrônicos para mediação da conexão e informações. A importância da relação do ambiente WEB com o ambiente móvel ( conexão Móbile-WEB) representa dois universos que se somam, apresentando uma cartografia lógica das informações a fim de proporcionar a comunicação e a garantia do processo fim-a-fim das informações e utilização remota por meio da Teleoperação radicalizada com o desenvolvimento da computação (pervasiva/ubiqua) a partir da popularização dos telefones celulares pelas redes caseiras (WIFI e WIMAX) ou de proximidade com a tecnologia Bluetooth.

Um exemplo nessa direção é o serviço Eprint que possui a funcionalidade de transformar imagens, documentos e tudo o que for digital, ou seja, bits, numa representação materializada em papel, instantaneamente, em qualquer lugar do mundo, acessível por um comando do celular. Então, as "coisas" estão começando a "conversar" com outras coisas à distância, como imprimir um documento no celular em qualquer lugar destino como clicar para enviar um email. Sem mesmo a impressora estar perto e nem no mesmo continente da impressora pela qual o seu arquivo será materializado. Por exemplo, se você está em São Paulo e precisar enviar um documento para a filial no Rio, não precisa colocar uma folha de papel dentro de um envelope do correio e nem enviar um email com o arquivo anexado para alguém imprimir. Simplesmente manda-se imprimir o documento na impressora do seu destinatário, dentre outros serviços. Sem a necessidade de um desktop ou o notebook, porque esse comando pode ser acionado a partir do seu celular [GETSCHKO,2009].

$\underline{\text { Mobilidade }}$

A mobilidade representa o estado de constante conexão em movimento por meio da utilização dos dispositivos, proporcionando uma nova relação entre as pessoas e com outros dispositivos fixos ou móveis. Essa possibilidade de transmitir informações sem a necessidade de um ponto fixo de conexão, e em movimento, transformou a colaboração e a interoperabilidade das informações.

A popularização em escala mundial dos aparelhos sem fio expressa a necessidade 
latente da sociedade em relação à difusão das informações conectado em movimento com a rede Internet. Smart Mobs é o termo criado por H.Rheingold (2002) para representar as novas práticas de utilização das tecnologias móveis como os celulares e Tablets por meio das mídias apresentadas nas formas de SMS, MMS, Blogs, Sites, Games, Videoconferência, etc. $O$ que traduz o enquadramento das relações humanas no cenário das soluções sistêmicas que fazem uso da mediação dos dispositivos móveis. A ideia da presença mediada aborda o contexto das interações no meio digital, apresentando os fenômenos inter-relacionados da teleoperação e telepresença. A Teleoperação para representar a intervenção real com ações efetuadas remotamente. Exemplos atuais que já podemos perceber com operações médicas efetuadas remotamente e robôs para análise de ambientes ermos com difícil acesso ao ser humano. A Telepresença com o objetivo de simular a permanência de um indivíduo sem a necessidade da transformação ou intervenção física do meio, como o exemplo da comunicação de voz mediado pela presença dos dispositivos sem fio [LEAO,2004]. A possibilidade de movimentação ou ação remota, por parte do usuário WEB, em outro ambiente físico, é reconhecida por alguns autores como "teleoperation" e implica na existência de algum dispositivo remoto mediando esta relação. Para Manovich (2001) "the essence of telepresence is that antipresence", pois uma atuação remota é capaz de afetar outras localidades sem a necessidade da presença física do participante nestes mesmos ambientes.

A Mobilidade caracteriza esta emergência dos desdobramentos sóciotecnológicos por meio da tecnologia móvel integrado com ambiente da rede Internet. A dinâmica que conecta e corrobora a colonização dos dispositivos sem fio tem explicação na cultura das comunidades virtuais e do comportamento humano que é móvel pela sua própria natureza. A dinâmica dessa troca das informações já apresentada na Internet, agora se apresenta expandido para as redes mediado pelos aparelhos móveis como celulares, smartphones e Tablets como alternativa para troca de informações em todas as áreas de conhecimento. Esse avanço tecnológico-social cada vez mais frequente no cotidiano para facilitar a necessidade da comunicação entre indivíduos que paralelamente ao avanço tecnológico das redes móveis, esse movimento social se molda para absorver a novas tecnologias e exercer a necessidade latente da relação e comunicação entre indivíduos e interferência no meio.

O desdobramento da ideia de uma multidão conectada pela mediação dos dispositivos móveis apresenta um cenário que transcende a necessidade de um espaço físico e fixo para comunicação; uma representação da conexão em movimento que está em permanente disposição para os usuários. Contexto que remonta a ideia de uma 
colonização do corpo pela tecnologia que se faz onipresente. A inclusão social no ambiente móvel já se faz presente em cidades que fazem uso desta tendência tecnológica, onde podemos perceber a utilização das redes móveis subsidiadas pelo poder público em algumas cidades brasileiras a fim de que prover um ambiente de comunicação livre para a população.

Como é o caso do município de Piraí (RJ), da cidade de Parintins localizada em uma ilha no rio Amazonas e da pequena cidade paulista de Sud Menucci. Estes locais conseguiram disponibilizar acesso gratuito por meio da conexão sem _o para população. A utilização da tecnologia móvel pela população tem o objetivo de aprimorar ou facilitar uma ação do cotidiano, em conjunto com o design dos dispositivos e sistemas que acompanham na mesma proporção o fenômeno da mobilidade para de estreitar o contato com o usuário e a ergonomia na interação com os dispositivos. Algumas análises arriscam em prever que haverá num curto prazo uma adoção de algum tipo de WearComp ${ }^{2}$. Dispositivos móveis que proporcionam desde um simples envio de mensagem até como forma de efetuar transações financeiras nos moldes de um cartão de crédito ou débito, para pagamento instantâneo de contas em postos de gasolina e lanchonetes

McDonalds

\footnotetext{
2 Termo para representar os computadores "vestíveis" como relógio e óculos representados pelas funcionalidades dos dispositivos móveis (LEÃO, Lúcia: Derivas. 2004, p.203)
}

[RHEINGOLD,2002]. Alguns exemplos no Brasil apresentam soluções de negócio com a utilização da tecnologia móvel como sinais da tecnologia da informação no meio móvel. Algumas iniciativas privadas estão lançando produtos para viabilizar pagamentos de contas em restaurantes, táxi, cinemas e supermercados como alternativa de integração com as administradoras de cartões de crédito e débito. A intenção é que a utilização do pagamento por meio de aplicativos embarcados no celular sirva de alternativa para os cartões de plástico.

\subsection{Tecnologia}

\section{Computação Pervasiva/Ubiqua}

A computação pervasiva é o conceito de uma solução com objetivo de prover a interoperabilidade das informações por meio da utilização dos Sistemas Computacionais implementados nos ambientes móveis concebidos para serem executadas em diversos dispositivos eletrônicos e preparados para desenvolver a comunicação e o processamento das informações com outros ambientes como a Internet, computadores PC (Personal Computer), e outros dispositivos eletrônicos locais e remotos e serviços disponíveis num ambiente (Ex. Redes WiFi, leitura de QRcode, Redes Bluetooth, etc) (HANSMANN, MERK e STOBER, 2003:1724).

O objetivo da computação pervasiva é permitir ao usuário o acesso à informação em qualquer local e aparelho de uma maneira 
personalizada para receber e solicitar as informações por meio de um dispositivo portátil de modo a transferir o computador da mesa de trabalho para um contexto móvel e descentralizado, o que Mark Weiser (1991,94104) chamou de computação invisível.

A mobilidade e o reconhecimento do contexto são dois aspectos importantes que representam características dinâmicas da computação pervasiva. $\mathrm{O}$ aspecto técnico da mobilidade se dá pela necessidade da permanência da conexão e da situação de contorno quando não há conectividade devido à ausência de sinal da comunicação da rede sem fio. O outro aspecto se dá pela percepção do contexto pelo qual se dá pelo reconhecimento dos estímulos do meio a fim de identificar e trocar informações com outros dispositivos [ILYAS e MAHGOUG, 2005: 53-59].

A computação ubíqua/pervasiva ou sensiente não representa somente "em todo Lugar", mas também "em todas as coisas" que implica numa colonização das ações do cotidiano pela tecnologia sem a necessidade de uma abstração de um teclado, "mouse", ou uma interface gráfica [GREENFIEL,2006:11, 48, 98, 101]. Segundo Mark Weiser a ideia de se colocar o aparato computacional como pano de fundo, concentrando-se na relação homem-homem e menos na interface homemmáquina.

Uma nova concepção dos computadores tem sido criada de modo a concentrar a solução sistêmica na integração com os estímulos do ambiente, numa alusão à computação invisível ao ser humano.

O ambiente pervasivo/ubiquo é uma nova área de pesquisa que está sendo considerada a terceira onda da computação. Originou-se da proposta de Mark Weiser, chamada Computação Ubíqua ou Sensiente (Ubiquitous Computing), também denominada Tecnologia Tranqüila (Calm Tecnologia), Computação Pró-Ativa (Proactive Computing) ou Computação Invisível (Invísible Computing). Mas o termo predominante é Computação Pervasiva. O termo pervasivo (espalhado, integrado, universal) não existe uma representação direta na língua portuguesa. Alguns autores consideram como sinônimo o termo Computação Ubíqua, pois este existe na língua portuguesa como onipresente. Porém outros autores consideram que a Computação Ubíqua está diretamente ligada a proposta de Weiser: a computação que está em "background", enquanto que a computação Pervasiva está mais associada com a integração e a interface (apud BIGONHA e GARCIA ,2006: 11).

\section{Computação Móvel}

A computação móvel se caracteriza pelo construto sistêmico que utiliza as redes móveis como ambiente de interoperabilidade das informações. As propriedades técnicas dessas soluções sistêmicas se enquadram no conceito da computação Pervasiva e Ubiqua. Essas aplicações móveis aderem tanto a uma 
arquitetura assimétrica P2A (Ponto a Aplicação) como representação da arquitetura da informação oriunda de um site responsável pela centralização das requisições oriundo de um ponto (o dispositivo ou PC) por meio de uma aplicação (software). Por outro lado uma arquitetura P2P (Ponto a Ponto) é representada pelas aplicações (software) distribuídas na rede, compartilhando de parte de seus recursos (impressoras, armazenamento e processamento) e serviços (compartilhamento de arquivos, aplicativos e banda de rede). Onde cada ponto se caracteriza tanto como provedor quanto cliente para colaboração mútua de informações sem, obrigatoriamente, passar por nenhuma entidade intermediária que se conectam e trocam informações com outros sistemas e apresentam boa parte das soluções instalada nos dispositivos móveis. As informações são integradas por meio dos protocolos, traduzidos pelos serviços computacionais embarcados nos dispositivos, que encapsulam as informações que são transmitidas por toda interface até o destino.

$$
\text { O esquema (Fig.1) apresenta a }
$$
abrangência do ambiente da computação descentralizada, tanto no conceito da arquitetura distribuída da informação desde o poder de interoperabilidade do transporte dos protocolos, passando pelo ambiente de infraestrutura de telecomunicação até chegar aos dispositivos móveis onde são exercidas as operações das aplicações pelos usuários. O que representa um cenário considerado por alguns autores como uma nova era da computação (ILYAS e MAHGOUB,2004) que trouxe um conceito do interoperabilidade mais amplo para o desenvolvimento de sistemas.

Figura 1: Visão geral da Interoperabilidade (Hansman,2003).

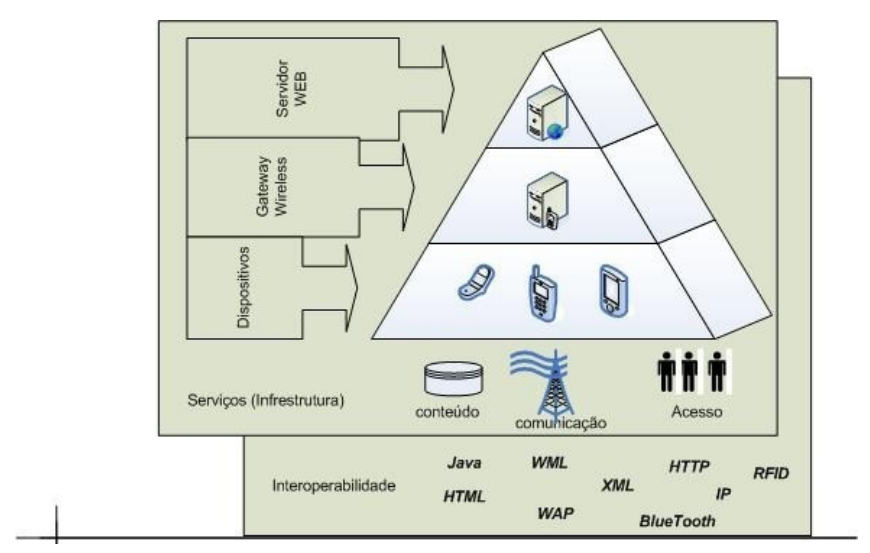

Como podemos perceber no histórico dos ambientes de integração (Fig.2) apresentado inicialmente pela centralização das informações na era dos Mainframes, dando-se posteriormente a distribuição pelos adventos das redes $\mathrm{LANs}^{3}$ e finalmente os computadores pessoais e celulares com acesso à Internet por meio de um ponto fixo ou sem fio, conexão com sensores e outros dispositivos.

A emergência da computação móvel, caracterizada pelos serviços proporcionados pelo ambiente de conexão dos diversos dispositivos (Fig.2), e sob o conceito da computação pervasiva/ubíqua, permite a captação dos estímulos dos outros

\footnotetext{
3 Rede local ou LAN - representa uma rede física de computadores interligados com cobertura limitada (geralmente limitado a um prédio, residência ou empresa).
} 
dispositivos eletrônicosinstalados no ambiente em que o usuário esteja fisicamente no local naquele momento, ou remotamente por meio da Teleoperação proporcionado pela interoperabilidade da rede.

Figura 2: Histórico do ambiente da Interoperabilidade (Hansman, 2003)

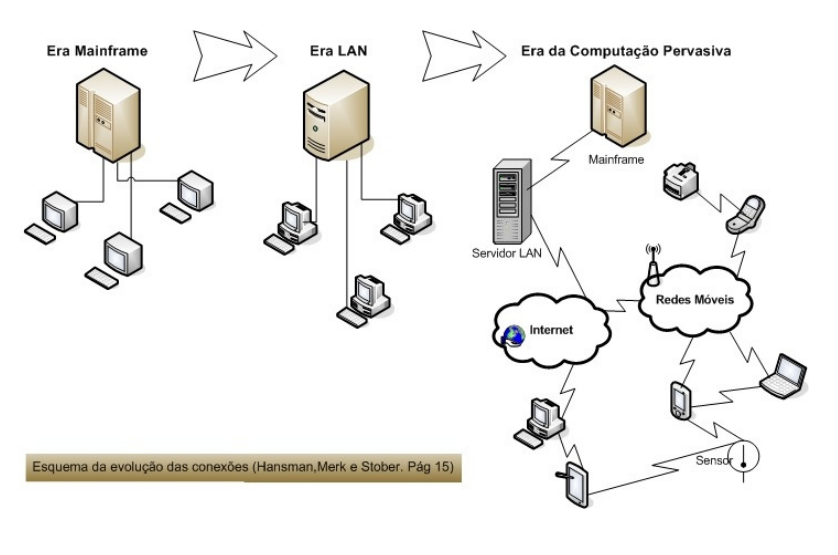

Portanto, o conceito do desenvolvimento de sistemas no meio móvel não está exclusivamente relacionado com ao acesso às informações no ambiente da Internet por meio da conexão Móbile-WEB, mas também às soluções móveis que já nascem com a concepção de aderência às limitações de tela e ao processamento dos dispositivos móveis em conjunto com a capacidade de identificação de estímulos e serviços disponíveis para serem consumidos no ambiente pelos usuários. Sobretudo, apresentam características a portabilidade, facilidade de uso, relativo baixo custo, e ampla disponibilidade de serviços móveis enquadrados ao perfil do usuário e aderentes às funcionalidades do cotidiano que vão muito além do simples serviço de voz, com a troca de serviços e informações com outros ambientes.

\section{Características Gerais das redes Móveis}

A tecnologia utilizada nas redes móveis permite a conexão dos aparelhos eletrônicos conectados por meio da tecnologia das Redes Moveis (UWB, BlueTooth, 3G, 4G, Wifi, WiMax, WiMesh e WAN).

O desenvolvimento da tecnologia das Redes Móveis promoveu composto de quatro principais generalizações PAN (Pervasive Network), LAN (Local area Network), MAN (Metropolitan Area Network) e WAN (Wide área network) de acordo com o alcance das ondas ou sinais e características técnicas de cada rede, ilustrados nos 4 segmentos a seguir.

Figura 3: Esquema das Redes Móveis e conexões (Autor,2014).

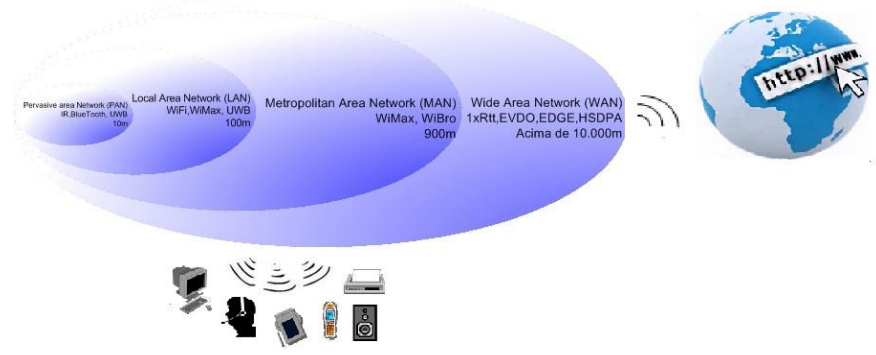

No primeiro segmento, a rede PAN (Pervasive Área Network) trata da conexão com alcance reduzido de alcance. Algumas configurações avançadas podem chegar a dezenas de metros, mas geralmente apresentam uma conexão de pequeno porte até 10 metros. Nas redes de um ambiente pervasivo há duas estratégias de utilização 
pelas aplicações móveis: uma com arquitetura baseada em pontos móveis de acesso que se ligam aos sites com as informações centralizadas, refletindo um modelo $\mathrm{P} 2 \mathrm{~A}$ (ponto a aplicação) na qual uma aplicação está localizada em outro site com alta dependência dos dados e serviços externos ao ambiente do dispositivo eletrônico móvel.

Outra estratégia se refere às redes dinâmicas móveis que remontam o conceito de uma rede ponto a ponto (P2P) com aplicações com baixo grau de dependência dos sites e mais autônomas aderentes à descentralização das informações e mais relacionadas à comunicação direta com outros dispositivos ou serviços disponíveis no ambiente. As características de comunicação em rede mais conhecidas e utilizadas da categoria das Redes PAN (Pervasive Área Network) estão representadas nas tecnologias BlueTooth (tipo de conexão utilizado em dispositivos portáteis e móveis), IrDA (Infravermelho), RFID (RadioFrequency Identi_cations) e o UWB (Ultrawideband) .

A conexão Bluetooth é uma tecnologia de transmissão de dados sem fio criada para conexão entre aparelhos próximos situados a uma distância de até 10 metros, como câmeras digitais, celulares, headsets, impressoras, teclados, mouses, etc. A taxa de transferência de dados alcançada com Bluetooth 1.0 é relativamente baixa (até 1Mbps) e sua área de cobertura é limitada em 10 (dez) metros na maioria dos casos. O Bluetooth 2.0 consegue transferir dados a 12 Mbps numa área com 10 a 20 metros de raio com a possibilidade de gerenciar operar $10 \quad$ conexões simultaneamente. A conexão RFID utiliza a tecnologia de transmissão por meio de ondas de rádio, que torna possível o rastreamento, identificação e troca de informação com outros dispositivos que também possuem a mesma interface. A identificação por rádio frequência é uma tecnologia que possui objetivo e utilidade similar ao código de barras.

O sistema de funcionamento RFID é composto por três elementos: uma antena, um leitor (similar ao do caixa de um supermercado) e um transmissor (correspondente à etiqueta do produto a ser identificado). A antena, localizada na etiqueta, recebe os sinais eletromagnéticos emitidos pelo leitor e, por sua vez, envia as informações que possui armazenada. Diferente dos códigos de barras convencionais, que exigem um contato direto entre o código impresso e o leitor (scanner), o sistema via sinais eletromagnéticos possibilita essa identificação à distância. Ambas enquadradas na tecnologia UWB (UltraWideband) $)^{4}$.

Inserido também nas redes PAN's, o IrDA (infravermelho) são desenvolvidos para

\footnotetext{
${ }^{4}$ UWB (Ultra Wide Band) - tecnologia de transmissão de dados conhecida como carrier-free, baseband ou impulse technology capaz de altas taxas de transmissão, transceptores de baixo custo, baixa potência de transmissão e baixa interferência.
} 
transmitir e receber dados por meio de pulsos de luz de infravermelho. A grande maioria dos dispositivos móveis possui uma porta de infravermelho incorporada para esse tipo de comunicação de dados. As velocidades típicas para a comunicação IrDA são de aproximadamente 4 Mbps que suprem a necessidade de comunicação fácil e rápida de dados entre aparelhos eletrônicos. $\mathrm{O}$ alcance da tecnologia IrDA é de, aproximadamente, apenas um metro. Isso é suficiente para transmitir dados de um notebook, tablets, smartphones, ou entre impressoras, mas não é recomendado para conexão em rede e/ou conexões simultâneas devido às restrições de alcance e taxa de transmissão.

No segundo segmento, as redes LAN's (Local Área Network) tratam da conexão com alcance até 100 metros, geralmente utilizada para ambientes internos, com a proposta de conexão para acesso à Internet por meio de ondas semelhantes a de rádio, que podem ser enviadas pelas tecnologias WiFi. Designação técnica também conhecida como Rede 802.11 pelo Institute of Electrical and Electronics Engineers (Instituto de Engenheiros Elétricos e Eletrônicos-IEEE) que estabelece padrões para uma variedade de protocolos eletrônicos de transmissão de dados em rede, dentre outras tecnologias de rede segmentadas por maiores capacidades de alcance e latência de transmissão como WiMax (Interoperabilidade Mundial para Acesso de Microondas),
WiMesh (Rede em Malha Sem Fio) e MóbileFi.

No terceiro segmento, as redes MAN's (Metropolitan Área Network), é versão mais abrangente com maior capacidade de transmissão e cobertura apropriada para cobertura de grandes áreas como cidades e municípios nas freqüências de 2,5Ghz, 3,5Ghz, 10,5Ghz e 5,8 Ghz (livre), conforme definido pelo entidade reguladora ANATEL (Agência Nacional de Telecomunicações) ${ }^{5}$. Como uma evolução do grupo da rede MAN podemos relacionar as tecnologias WiMesh e MobileFi.

A tecnologia WiMesh utiliza os sistemas sem fio das redes WiFi/WiMax. A infraestrutura da rede WiMesh é composta por várias antenas com capacidade de cobertura menor que o WiMax e maior que o WiFi. De modo que a infraestrutura das antenas se apresentam mais próximas umas das outras e tornam a transmissão mais densa e segura, com velocidades de 54 Mbps e alcance de até 400 metros.

A rede MobileFi apresenta o pioneirismo na utilização de um padrão especificamente projetado para carregar o tráfego IP nativo para acesso em banda larga de forma completamente móvel. Ele proporciona taxas de transmissão simétricas de $1 \mathrm{Mbps}$ a $4 \mathrm{Mbps}$ em espectros licenciados abaixo de 3,5 GHZ em distâncias de 15 km aproximadamente. Isto faz com que a rede

\footnotetext{
${ }^{5}$ Órgão vinculado ao Ministério das Comunicações para regulação dos serviços de voz e dados no Brasil.
} 
MóbileFi tenha menos potência que o WiMax, mas com a característica da implementação de um protocolo móvel nativo com características técnicas de latência até, que possibilita grande estabilidade de conexão até mesmo para um veículo movimentando-se rapidamente.

No quarto e último segmento das redes sem fio, as redes WAN's (Wide Área network) representam a evolução dos padrões da telefonia celulares conhecidas no mercado como a terceira $(3 \mathrm{G})$ e quarta geração $(4 \mathrm{G}) \mathrm{da}$ telefonia celular, com velocidade de transmissão de 2Mbps até 10Mbps e conceituada tecnicamente na categoria UMTS (Universal Móbile Telecomunication System $)^{6}$ também utilizada

no terceiro segmento, que sucederá a categoria GPRS (General Package Radio

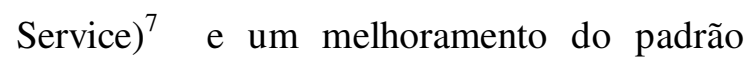
UMTS baseada na tecnologia GSM (Global System Mobile) com velocidades de transmissão de até $384 \mathrm{Kbps}$.

Atualmente a tecnologia de transmissão GSM é utilizada no Brasil pelas principais operadoras: VIVO, Oi, Tim, Claro. O avanço

\footnotetext{
${ }^{6}$ UMTS - Tecnologia de transporte de informações de alto desempenho considerada superior ao GPRS com serviços de roaming universal, transporte de aplicações multimídia, convergência de acesos e conexão com a internet com velocidades até $2 \mathrm{Gbps}$ (TALUDKER, YAVAGAL,2007:281282).

${ }^{7}$ GPRS - Tecnologia de transporte de informações de alto desempenho para a rede sem_o .

Utilizada inicialmente na infraestrutura das redes GSM e TDMA. Apresentou-se na época como

uma solução satisfatória com velocidades de transmissão que alcançava 141,4 KBps a $384 \mathrm{KBps}$

(TALUDKER, YAVAGAL,2007:203-204).
}

da tecnologia móvel representa um alto desempenho na transmissão de voz e dados com maiores velocidades e, consequentemente, maiores ofertas de serviços como vídeo, TV, Internet e download/upload mais rápidos, dentre muitas outras vantagens, com relação à interoperabilidade das informações baseado no protocolo IP (Internet Protocol) através das principais gerações de tecnologias de redes (Fig.4) como a de terceira geração $3 \mathrm{G}$ (WCDMA, HSDPA, EVDO), e da quarta geração 4G (LTE) atualmente ainda em processo de implementação no mercado brasileiro.

Figura 4: Geração das Redes Móveis (Talukder,2007).

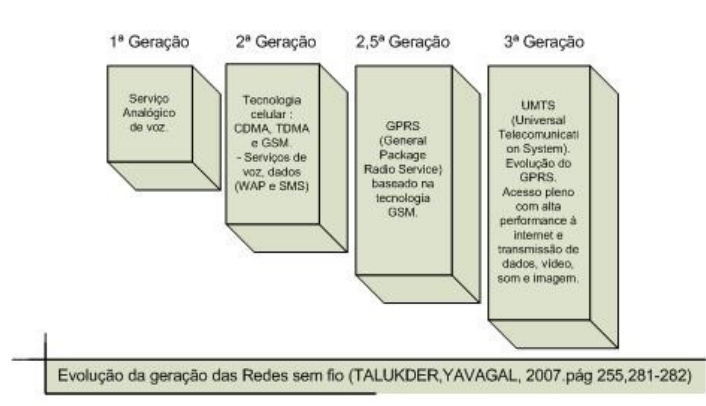

$\underline{\text { Arquitetura de Serviço Móvel (MOA) }}$

Os seguintes questionamentos são apropriados devido ao crescente apelo das soluções móveis do momento atual:

- As arquiteturas sistêmicas móveis são escaláveis, ou seja, atendem aos anseios de integração com as informações existentes em diversas plataformas? 
- Há tempo de resposta aceitável das informações, considerando o volume crescente de acesso pelos usuários ? Como lidar com a integração da diversidade dos ambientes e dispositivos lançados no mercado?

Em resposta aos questionamentos acima, o termo MOA (Móbile Oriented Architecture) acrônimo apresentado por $\operatorname{PATRONE}(2006)^{8}$ representa arquitetura de serviços distribuídos no ambiente heterogêneo móvel com o objetivo de implementar a transparência e disponibilidade dos acessos aos serviços disponíveis pelas operadoras de celulares (ex. VIVO, CLARO, TIM) e consequentemente do consumo desses serviços pelos clientes/usuários que relaciona a idéia da arquitetura de negócio SOA para modelagem de negócio orientada a serviço (Service Oriented Architecture) como uma referencia da utilização dos Web Services que utilizam os protocolos SOAP, UDDI e WSDL como uma tecnologia de integração sistêmica na rede Internet com objetivo de elucidar a interoperabilidade das informações por meio de serviços sistêmicos que podem ser publicados e consumidos numa plataforma distribuída.

O princípio da arquitetura orientada a serviço móvel pode ser considerada no mesmo conceito da arquitetura SOA como abordagem para disponibilidade dos serviços

${ }^{8}$ PATRONE, Dennis. Toward na Architecture for Móbile Object in Heterogêneos Distribuited Enviroments, 2006:3). Dennis . S. Patrone is a Ph. D. student at the State University of New York, University at Bufalo, senior professional sta Hopkins University / Applied Physics Laboratory in Laurel, MD. móveis (SMS, Redes sem fio, Voz, Dados, Vídeo Conferencia, etc) que, dentre outras soluções, também permitem o acesso e a troca de informações dos Web Services por meio da conexão Mobile-WEB pelos dispositivos descentralizados.

O esquema abstrato (Fig.5) apresenta o ambiente genérico de conectividade da arquitetura pervasiva com os atores de integração Móbile-WEB e dispositivos do ambiente local.

Figura 5: Conexão do ambiente Pervasivo dos serviços móveis (JIN,2006).

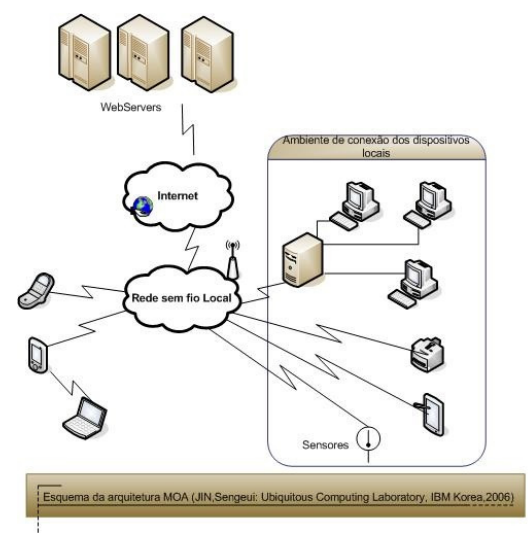

A tecnologia para o desenvolvimento de sistemas é um meio para o acesso às funcionalidades dos aparelhos eletrônicos disponíveis pelos fabricantes de celulares e de acesso às interfaces com os serviços oferecidos pelas operadoras de telecomunicações.

As alternativas para 0 desenvolvimento de sistemas móveis apresentam ambientes sistêmicos distintos de acordo com a proposta dos fabricantes. Porém, ainda não há um critério 
determinístico sobre a melhor plataforma de mercado para o desenvolvimento das mídias móveis devido ao número das alternativas de soluções e possibilidades técnicas disponíveis.

Dessa forma as soluções móveis dispõem de um ambiente fértil para o enquadramento no modelo de negócio mais conveniente, conforme a gama de produtos já existentes para definir os rumos do seu próprio sucesso, moldado em torno dos desejos de seus consumidores, regras de negócio e necessidades técnicas, distribuído numa região ou ambiente informacional restrito.

\section{Resultado}

O resultado desse artigo é contextualizar o conjunto das informações multidisciplinares direcionado aos profissionais do mercado, pesquisador, estudioso ou usuário do meio digital envolvido direta ou indiretamente com o processo de inovação dos produtos e serviços no meio digital intitulada Internet das Coisas. Aspecto evidenciado no discorrer dos assuntos abordados pela interoperabilidade do ambiente móvel para o desenvolvimento de soluções sistêmicas quando percebemos a relação do individuo com a tecnologia móvel no cotidiano da sociedade e nos conscientizamos, por meio dos assuntos abordados, a intensidade dessa relação e da dependência da necessidade da informação em tempo real para, eventualmente, decidir a próxima ação do nosso cotidiano como precaver-se de um tráfego lento a caminho de casa; ou a identificação de uma rota ou localização de um destino; ou enviar informações "On Line" de um acontecimento que mereça notoriedade; ou até mesmo a veicular informação de um imprevisto que justifique um atraso durante o percurso ao destino; até mesmo criar redes de informações para mobilizar um grupo de pessoas para qualquer tipo de evento, veicular informações segmentadas ao publico alvo, e criar "Hubs"' de informação.

As observações sobre o ambiente informacional da Internet das Coisas adotam uma orientação baseada na aplicação das diretrizes do trabalho pesquisa [PAES,2013] que destaca o processo de Inovação como uma área de conhecimento complexa e multidisciplinar. A gestão tecnológica relaciona, dentre outras questões e princípios, a atuação dos profissionais envolvidos com desenvolvimento de produtos e serviços no meio digital, a necessidade de articularem outras áreas de conhecimento na análise e implementação das soluções sistêmicas.

É importante destacar que este referencial teórico-analítico não é somente estático, como também dinâmico e flexível, aplicado a cada ambiente informacional. Que na abordagem desse artigo considerou como foco de análise o ambiente intitulado Internet das Coisas como apresentado no desenvolvimento dos assuntos desse artigo.

\footnotetext{
${ }^{9}$ Em rede a ideia HUB é concentrar e ligam diversos outros nós caracterizado por outros usuários conectados, formando "grupos"segmentados em rede.
} 
Dessa forma, a Internet das Coisas relaciona a tecnologia disruptiva dos "objetos inteligentes" (dispositivos) inserida em grande escala no cotidiano da sociedade como tendência do mercado atualmente. Cenário que precisa ser considerado no processo de inovação, considerando a contribuição desse ambiente no crescimento da produção de dados/informações. A EMC Corporation, que, em parceria com a análise da International Data Corporation (IDC) $)^{10}$, quantificou a previsão do número de dispositivos conectados à internet está numa ordem de grandeza de 200 bilhões, com 7\% (14 bilhões) já funcionando ou se comunicando via web. Um ambiente de interoperabilidade proporcionará benefícios ao mercado das empresas de software e usuários devido ao alcance dos dispositivos, proporcionando novas dinâmicas de fluxos das informações e modelos de negócio. Representado pelo enquadramento do processo de inovação das empresas de software nesse novo ambiente sócio-tecnológico da Pervasão e Ubiquidade para o desenvolvimento dos seus produtos e serviços como grande desafio já considerada como a terceira onda da computação. O uso crescente e contínuo do uso de smartphones, redes móveis, computação móvel, internet e redes sociais; representam um ambiente fértil para Internet das Coisas ser considerada como

10

http://corporate.canaltech.com.br/noticia/mercado/Inter net-das-Coisas-impulsionaexpansaono-Universo-Digital/ixzz34U257Ewx - Creative Commons (CC BY-NC-ND). uma plataforma para o desenvolvimento de novos produtos e serviços.

Esse ambiente de interoperabilidade carrega também a compreensão de um ambiente multidisciplinar e distribuído com limitações e dificuldades de "interoperar" informações entre ambientes que não estão sob total controle do emissor, necessitando da colaboração, comunicação, interpretação e trafego das informações pelos atores do meio.

De modo que não existe um impedimento tecnológico nem um cenário de futurologia em relação à Internet das Coisas no que tange a consideração no processo de inovação dos produtos e serviços. Uma vez que as tecnologias necessárias já se encontram disponíveis de acordo com das alternativas tecnológicas apresentadas desse artigo, prontas serem utilizadas para enfrentar novos desafios. O que representa ao gestor a responsabilidade de articular as informações e tecnologias disponíveis e aplicadas num ambiente informacional a fim de criar novas soluções de negócio.

\section{Conclusão}

A emergência do meio móvel e da popularização acelerada dos dispositivos

que utilizam a tecnologia sem fio, representam um fator importante para a interoperabilidade das informações no ambiente da Internet das Coisas. A intensificação desse contexto nos conduz para uma reflexão sobre a influência e utilização das novas tecnologias de comunicação e 
colaboração das informações, além dos movimentos já conhecidos e proporcionados na Rede Internet como as Midias Sociais, Crowdsourcing, blogs, etc .

Entretanto, ao mesmo tempo, quando abordamos a emergência das novas tecnologias, é importante ressaltar o reflexo do fator sócio-tecnológico multidisciplinar. De modo que o aspecto do transporte e dinâmica das informações proporcionadas pelo aparato tecnológico, torna-se cada vez mais influente no cotidiano da sociedade. Isso, por um lado, proporciona uma visão inovadora e desenvolvimentista, mas por outro lado uma visão de interdependência crescente da tecnologia disponível num ambiente para consumo pela sociedade que proporciona um mercado consumidor para comercialização dos produtos e serviços no meio digital.

Esse contexto sócio-tecnológico das tecnologias "invisíveis" (Pervasiva/Ubiqua) se torna cada vez mais amplo para análise e objeto de estudo da influencia da tecnologia sem fio na sociedade. Uma relação cada vez mais intensa e próxima à medida que o fator humano representa um fator preponderante do processo de inovação, criando novas necessidades de integração e relacionamento que dependem da tecnologia como mediadora. Dessa forma, a intensificação e abrangência do móvel abordados neste artigo nos fornecem subsídios para uma reflexão sobre consequências e cenários que sem dúvida se apresentam inovadores e cada vez mais aderentes ao comportamento natural do ser humano. Uma vez que esse usuário está incorporado nesse sistema Cíbrido caracterizado pelo papel ativo e ampliado da presença humana pela utilização dos dispositivos para além dos limites de sua forma material quando interage com outro individuo ou meio (dispositivo, servidor de Software, e infraestrutura das Redes Móveis), tornando-o cada vez mais influente num espectro tecnológico abrangente para interferir nos negócios e nas relações humanas.

Essa nova relação "homem-maquinahomem" por meio da tecnologia visa proporcionar facilidades para saciar os novos desejos de consumo. Quando muitas vezes não há percepção de muitos profissionais de tecnologia/sistemas e áreas correlatas, responsáveis pela inovação de seus produtos e serviços, que o comportamento do seu público está mudando sob influência das facilidades e disponibilidades tecnológicas oriundas de outras áreas de conhecimento. E que precisam estar relacionadas na concepção dessas soluções com objetivo de atender as exigências da sociedade moderna. Dessa forma a inovação tecnológica se faz presente e caracterizada pelo processo de desenvolvimento das novas ferramentas aplicadas para integração das informações e ações no ambiente digital de forma interdisciplinar e multidisciplinar com objetivo de acompanhar as novas 
necessidades da sociedade contemporânea nas

suas relações comerciais e culturais.

\section{Referências Bibliográficas}

ANTOUN,H. O Poder da Comunicação e o Jogo das Parcerias na Cibercultura, 2004.

BURBECK,S. Applications Programming in Smalltalk-80(TM): How to use Model-ViewController (MVC). 1992. Disponível em: http://stwww.cs.uiuc.edu/users/smarch/stdocs/mvc.html. Acesso em: 7/01/2008.

GETSCHKO, Demi. Internet, Mudança ou Transformação?. Artigo Nick.br, I septiembre09 de junho de 2009 । http://www.cgi.br/publicacoes/artigos/artigo5 9.htm.

GETSCHKO,Demi. Apresentação no programa Roda Viva https://www.youtube.com/watch?v=15sV0cIPC8 . São Paulo: TV Cultura,

Segunda Feira 13 de março 2009, acessado em 15/Nov/2012.

GETSCHKO,Demi. Apresentação Observatório da Imprensa: São Paulo, Quarta-Feira, 11 março 2009 _ ISSN 15197670 _ Ano 13 _ No. 435 31/3/2009.

GREENFIELD,Adam. Everyware: The dawning age of ubiquitous Computing,

AIGA, 2006, cap. 2,4,5.

HANSMANN,Uwe, MERK, Lothar; STOBER, Thomas; NICKLOUS, Martin. Pervasive Computing, Springer ,2003,pg. 64.

HORAM,Andy. Peer-to-Peer: O Poder transformador das redes ponto a ponto., ORELLY-Champbel, Berkley, 2001, Cap.1-4.

JIN, Sengeui. Ubiquitous Computing Laboratory, IBM, Korea, 2006.
RHEINGOLD, H. SmartMobs,2002, p.112

RISCHPATER, Ray. Desenvolvendo Wireless para WEB - Como enfrentar os desafios dos projetos para WEB sem _o, Cap. 1 p.8-15, p.129-172.

SCHILLING, Melissa, L. Strategic Management of Technological Innovation Third edition. NY University: McGraw-Hill, 2010:50-181.

SANTAELLA, Lucia. Linguagens Líquidas na era da mobilidade, 2007, p.24, p.194.

TALUKDER, Asoke, YAVAGAL, Roopa. Móbile Computing _ $\quad$ Technology, Application

and Service Creation, McGraw-Hill Communication Engineering,2007.

WEISER, M. The Origins of Ubiquitous Computing Research at PARC in the late 1980s - IBM Systems Journal,1999. pág 38,4, 1997.

PAES, Wander. Interoperabilidade dos Dispositivos Móveis. São Paulo: PUC SP, 2008. 100 f. Tese (Mestrado) Departamento de Pós-Graduação TIDD Tecnologias da Inteligência e Design Digital, Faculdade de Tecnologia e Mídias, PUC/SP, 2008.

PAES, Wander. Integracion Colaboracion em El Ciberspacio. Revista de Filosofia e Ciência, ISSN 1852-9488, Año II I número 5 | septiembre-diciembre de

2011 I www.prometeica.com.ar, 2011:3-7, 813.

PAES, Wander. Artigo Management Technology. 10 CONTECSI International

Conference o Information System and Technology Management - FEA USP, DOI:10.5748/9788599693094,12 a 14 de junho 2013 - pg 151.

LEÃO,L. DERIVAS: Cartografia do Ciberespaço, Annabluma, 2004, Cap. 1,3,5 